01

\title{
Определение концентрации компонентного состава газов методом оптической спектроскопии в условиях перекрытия их спектральных линий
}

\author{
(C) М.М. Кугейко, А.А. Баравик
}

Белорусский государственный университет, 220030 Минск, Республика Беларусь

e-mail: kugeiko@bsu.by

Поступила в редакцию 16.02.2021 г.

В окончательной редакции 16.02.2021 г.

Принята к публикации 05.07.2021 г.

Исследована эффективность определения концентрации компонентного состава газовых сред методом оптической спектроскопии на основе использования регрессионных соотношений, устанавливаемых между определяемыми и измеряемыми параметрами. Показаны возможности оперативного определения количественных значений концентраций исследуемых компонент газовой среды, повышения точности. Оценена эффективность использования данного подхода в условиях сильного перекрытия спектральных линий газовых компонент.

Ключевые слова: многокомпонентные газовые среды, концентрация компонентного состава, перекрытие спектральных линий, поглощение, главные компоненты, связь главных компонент с поглощением.

DOI: $10.21883 /$ OS.2021.10.51485.1919-21

\section{Введение}

Существует множество методов газового анализа: механические, акустические, тепловые, магнитные, электрохимические, полупроводниковые, массспектрометрические, оптические. Все эти методы, как правило, требуют отбора проб. А это и сложно, и приводит к дополнительным погрешностям определяемых величин. Они используются в основном для однокомпонентных и бинарных газовых сред [1].

Из оптических методов на практике наибольшее применение получили абсорбционные, базирующиеся на измерении ослабления излучения контролируемой средой. Эти методы, в свою очередь, подразделяются на дисперсионные, требующие спектрального разложения посылаемого излучения и регистрации спектров поглощения, и недисперсионные, в которых измеряется разность световых потоков, пропущенных через исследуемую среду и нейтральный ослабитель. Недостатком данных методов является сильная зависимость от изменений аппаратурных констант приемно-излучающих, регистрирующих блоков измерительной системы, температуры, давления окружающей среды. Они требуют использования узкополосных фильтров, высокомонохроматических источников излучения, защиты оптических элементов от загрязнений [1].

С появлением лазеров данные методы получили дополнительные преимущества, поскольку им присущи такие достоинства как высокая концентрационная чувствительность, оперативность, дистанционность, высокое пространственное разрешение. Особо следует выделить среди них метод дифференциального поглоще- ния [2]. Он широко используется в средах с возможностью выделения центра и крыла линий поглощения газовых компонент.

Для многокомпонентных сред при перекрытии их спектральных линий используются многоспектральные измерения (устанавливаются комбинации длин волн [3-6]), требующие решения обратной задачи. При этом задача нахождения концентрации газовых компонент из результатов измерений относится к классу некорректных математических, поскольку решение таких задач может быть неустойчивым по отношениям к малым вариациям (погрешностям) измеряемых данных, приводит к большим погрешностям в определении компонентного состава смеси [3,7].

Типично все алгоритмы решения обратных задач основаны на сравнении результатов измерения с функцией, описывающей связь регистрируемых сигналов с параметрами исследуемой среды, или же используют калибровочные модели, полученные заранее с использованием эмпирического (экспериментального) моделирования [4]. Находят такой набор параметров, при котором их различие будет минимальным. Как отмечено в [5], решения обратной задачи существенно зависят 1) от типа выбранной физической модели среды и приближений; 2) от объема и качества априорной информации; 3) от размерности вектора неизвестных параметров и измеренных значений; 4) от качества определения характеристик погрешности измерений. Указанные особенности решения, с одной стороны, позволяют получать соответствующего качества решения (оценки), однако эти оценки весьма затратны по времени при больших размерностях вектора измерений; существенно 
зависят от величины погрешности, могут при решении получать физически неверные результаты (обнуления, отрицательные значения и др.); требуют правильного и тщательного выбора физической модели и приближений [5].

Имея начальное приближение, решать обратную задачу можно известными методами Ньютона, НьютонаРафсона, Левенберга-Маркуарта и др. [6]. Однако при использовании этих методов необходимо оперировать большими спектроскопическими массивами данных. К тому же получаемое решение, как правило, включает много итераций. Все это снижает оперативность обработки экспериментальных данных и делает практически невозможным определение концентраций газов во время проведения эксперимента (режим реального времени) [6] .

Методы регуляризации Тихонова [7], используемые для решения некорректных обратных задач, требуют дополнительной информации по выбору параметра регуляризации, от установления которого зависит точность определяемых параметров. Однако в случае сильного зашумления данных, слабого поглощения газовых компонент используемая система уравнений является плохо обусловленной. Для нее даже метод регуляризации не гарантирует приемлемого решения [5-7].

Задача контроля многокомпонентных газовых сред еще больше усложняется, когда их спектральные линии сильно перекрываются. В этом случае использование выбранных спектральных каналов измерения, соответствующих линиям поглощения исследуемых компонент, требует учета вклада их перекрытия [8]. Если при этом проводить спектральные измерения при большом наборе спектральных каналов, то для решения данной задачи используются инверсные методы, комбинирующие математический алгоритм решения обратной задачи [9] и один из методов расчета переноса излучения в среде (наиболее точный из них - метод МонтеКарло [10]). Используемые численные методы расчета характеристик поля рассеянного или проходящего через среду излучения либо не обеспечивают необходимой для практики точности, либо требуют больших вычислительных затрат (как, например, метод МонтеКарло), и поэтому исключают возможность оперативной интерпретации спектральных измерений. Кроме того, для устойчивого решения обратной задачи необходимо, чтобы количество спектральных каналов регистрации излучения в несколько раз превышало количество модельных параметров [11-15], что еще больше затрудняет интерпретацию экспериментальных данных в режиме реального времени.

Ниже рассматривается возможность оперативного определения компонентного состава газовых смесей с перекрывающимися линиями их поглощения и повышения при этом точности измерений.

\section{Регрессионные соотношения между концентрациями газовых компонент и измеряемым спектральным поглощением}

Новый подход к решению обратной задачи оптического зондирования аэрозольных сред на основе установления регрессионных соотношений между определяемыми параметрами и измеряемыми оптическими характеристиками был предложен в [16]. Регрессионный метод решения обратных задач предполагает, что искомые параметры среды могут быть найдены при помощи некоторой статистической функции от измеряемых оптических характеристик. Получим ее для многокомпонентных газовых сред с сильно перекрывающимися спектральными линиями.

При прохождении излучения на посылаемых длинах волн происходит поглощение его компонентами газовой смеси. Величины сигналов для каждой из регистрируемых длин волн $P\left(\lambda_{i}\right)$ можно записать в следующем виде $[2,17]$ :

$$
P\left(\lambda_{i}\right)=A_{i} P_{0}\left(\lambda_{i}\right) \exp \left\{-\sum_{k=1}^{s} M_{k} \sigma_{k}\left(\lambda_{i}\right) l\right\},
$$

где $M_{k}-$ концентрации $k$-й газовой компоненты на длине волны $\lambda_{i} ; s$ - число газовых компонент в смеси; $\sigma_{k}\left(\lambda_{i}\right)$ - сечение поглощения соответствующих газовых компонент смеси; $l$ - длина измерительной трассы (или ячейки, если газ находится в оптической кювете); $P_{0}\left(\lambda_{i}\right)$ - энергия посылаемого излучения; $A_{i}$ - аппаратурная константа измерительной системы для каждой из длин волн $\lambda_{i}$.

Выражение (1) содержит аппаратурные константы $P_{0}\left(\lambda_{i}\right)$ и $A_{i}$, которые определяются измерением сигналов $P\left(\lambda_{i}\right)$, проходящих через участок среды длиной $l$, в отсутствие газовых компонент. Другими словами, данная процедура (в отсутствие газовых компонент) является калибровкой измерительной системы для получаемых величин поглощения.

В самом деле, при этом величины регистрируемых сигналов $P\left(\lambda_{i}\right)$ для каждой из длин волн $\lambda_{i}$ равны

$$
\begin{gathered}
P_{1}\left(\lambda_{i}\right)=A_{i} P_{0}\left(\lambda_{i}\right), \\
I\left(\lambda_{i}\right)=P\left(\lambda_{i}\right) / P_{1}\left(\lambda_{i}\right)=\exp \left\{-\sum_{k=1}^{s} M_{k} \sigma_{k}\left(\lambda_{i}\right) l\right\} .
\end{gathered}
$$

Будем оперировать сейчас отношением сигналов $I\left(\lambda_{i}\right)=P\left(\lambda_{i}\right) / P_{1}\left(\lambda_{i}\right)$, являющимся величиной поглощения, которое равно

$$
I\left(\lambda_{i}\right)=\exp \left\{-\sum_{k=1}^{s} M_{k} \sigma_{k}\left(\lambda_{i}\right) l\right\} .
$$


Для $I\left(\lambda_{i}\right)=P\left(\lambda_{i}\right) / P_{1}\left(\lambda_{i}\right)$, нормированного на энергию посылаемого излучения и аппаратурные константы, выражение (3) можно записать в следующем виде:

$$
\begin{aligned}
\ln I\left(\lambda_{1}\right) & =-\frac{1}{l}\left(M_{1} \sigma_{1}\left(\lambda_{1}\right)+M_{2} \sigma_{2}\left(\lambda_{1}\right)\right. \\
& \left.+M_{3} \sigma_{3}\left(\lambda_{1}\right)+\ldots+M_{s} \sigma_{s}\left(\lambda_{1}\right)\right), \\
\ln I\left(\lambda_{2}\right) & =-\frac{1}{l}\left(M_{1} \sigma_{1}\left(\lambda_{2}\right)+M_{2} \sigma_{2}\left(\lambda_{2}\right)\right. \\
& \left.+M_{3} \sigma_{3}\left(\lambda_{2}\right)+\ldots+M_{s} \sigma_{s}\left(\lambda_{2}\right)\right), \\
\ln I\left(\lambda_{n}\right) & =-\frac{1}{l}\left(M_{1} \sigma_{1}\left(\lambda_{n}\right)+M_{2} \sigma_{2}\left(\lambda_{n}\right)\right. \\
& \left.+M_{3} \sigma_{3}\left(\lambda_{n}\right)+\ldots+M_{s} \sigma_{s}\left(\lambda_{n}\right)\right) .
\end{aligned}
$$

Для интерпретации измерительной информации необходимо решить систему уравнений (4), которую можно записать в виде

$$
B \mathbf{x}=\mathbf{F},
$$

где

$$
\begin{aligned}
& B=\left(\begin{array}{cccc}
\sigma_{1}\left(\lambda_{1}\right) & \sigma_{2}\left(\lambda_{1}\right) & \ldots & \sigma_{1}\left(\lambda_{1}\right) \\
\sigma_{1}\left(\lambda_{2}\right) & \sigma_{2}\left(\lambda_{2}\right) & \ldots & \sigma_{1}\left(\lambda_{2}\right) \\
\ldots & \ldots & \ldots & \ldots \\
\sigma_{1}\left(\lambda_{n}\right) & \sigma_{2}\left(\lambda_{n}\right) & \ldots & \sigma_{1}\left(\lambda_{n}\right)
\end{array}\right) \\
& \mathbf{x}=\left(\begin{array}{c}
M_{1} \\
M_{2} \\
\ldots \\
M_{s}
\end{array}\right), \quad \mathbf{F}=-\frac{1}{l}\left(\begin{array}{c}
I\left(\lambda_{1}\right) \\
I\left(\lambda_{2}\right) \\
\ldots \\
I \lambda_{n}
\end{array}\right)
\end{aligned}
$$

$n$ - число длин волн.

Однако поскольку измеряемые величины поглощения и величины сечений поглощения определяются с погрешностями, то данная система относится к системе неоднородного вида. Эта система относится к классу некорректных обратных задач. Решение таких задач может быть неустойчивым по отношениям к малым вариациям (погрешностям) измеряемых данных. Это приводит к большим погрешностям в определении компонентного состава смеси [3-7].

Значения $I\left(\lambda_{i}\right)$ можно рассматривать как компоненты случайного вектора $\mathbf{I}\left(\lambda_{i}\right)$. Разложим вектор $\mathbf{I}\left(\lambda_{i}\right)$ по системе ортонормированных базисных векторов (главных компонент) $[16,18]$

$$
\mathbf{I}\left(\lambda_{i}\right)=\overline{I\left(\lambda_{i}\right)}+V C_{m},
$$

где $V$ - матрица со столбцами из главных компонент $C_{m}$, численные значения которых определяются как проекции вектора $\mathbf{I}\left(\lambda_{i}\right)$ на пространство из главных компонент и находятся следующим образом:

$$
C_{m}=V^{t}\left(\mathbf{I}\left(\lambda_{i}\right)-\overline{\mathbf{I}\left(\lambda_{i}\right)}\right)
$$

где индекс $t$ означает операцию транспонирования.

В связи с быстрой сходимостью рассматриваемого разложения на первые базисные векторы приходится большая часть изменчивости вектора $\mathbf{I}\left(\lambda_{i}\right)$, а соответствующие им главные компоненты содержат в себе практически столько информации, сколько ее было в исходных данных. Следовательно, для восстановления концентраций компонентного состава газовой смеси $M_{k}$ можно использовать не сам вектор измерений, а его главные компоненты.

С точки зрения оперативности определения концентраций компонентного состава газовой смеси представляет интерес возможность их расчета на основе аналитического выражения, связывающего их с главными компонентами вектора $\mathbf{I}\left(\lambda_{i}\right)$ для используемых длин волн $n$. Такое выражение может быть получено на основе измерений или численного расчета $I\left(\lambda_{i}\right)$ для множества ситуаций или моделирующих его фантомов с известными значениями концентраций $M_{k, P, T}$ компонентного состава газовой смеси при различных значениях ее температуры $T$ и давления $P$ (т.е. учитывается уширение спектральных линий с их изменением) и последующего регрессионного анализа, устанавливающего связь между $M_{k, P, T}$ и $C_{m}$. В частности, в качестве такого выражения можно использовать регрессионные соотношения

$$
M_{k, P, T}=a+\sum_{m=1}^{N} b_{m} C_{m},
$$

где $N$ - количество главных компонент.

Численные значения $a, b m$ определяются путем расчета по формуле (5) главных компонент $C_{m}$ для всех реализаций $I\left(\lambda_{i}\right)$, каждой из которых соответствует конкретное значение $M_{k, P, T}$, и использовании метода наименьших квадратов для аппроксимации статистической связи между $C_{m}$ и $M_{k, P, T}$.

После получения векторов $\mathbf{I}\left(\lambda_{i}\right)$ и среднего вектора $\mathbf{I}\left(\lambda_{i}\right), V$, а также вышеотмеченного аналитического выражения (6) они могут применяться для определения компонентного состава газовой смеси по результатам измерения спектрального поглощения. Численное значение $a, b_{m}, C_{m}$ можно устанавливать как для каждой пары значений давления $P$ и температуры $T$, так и для задаваемого диапазона их значений.

Таким образом, определение компонентного состава газовых смесей включает нахождение по формуле (5) главных компонент для величин спектрального поглощения и определение концентрации на основе регрессионного соотношения (6), соответствующего температуре и давлению (в смеси в момент проведения измерений) или же задаваемому диапазону их значений. При этом для интерпретации измерительной информации не требуется выполнения каких-либо условий или использования дополнительной информации о состоянии исследуемой среды, использования калибровочных сравнительных моделей, устанавливаемых заранее эмпирически (экспериментально), применения методов решения некорректных обратных задач. Появляется устойчивость к погрешностям определения величин поглощения (установление связей между концентрацией и измеряемыми 
параметрами осуществляется с учетом погрешностей измерительной аппаратуры).

\section{Оценка эффективности метода для задаваемых значений давления и температуры}

Рассмотрим более детально процесс получения главных компонент $C_{m}$ и коэффициентов регрессии $a, b m$ на примере определения концентраций следующих газовых компонент: паров воды $\mathrm{H}_{2} \mathrm{O}$ и углекислого газа $\mathrm{CO}_{2}$ в атмосфере. Это актуальная в настоящее время задача в связи с парниковым эффектом, с которым связывают

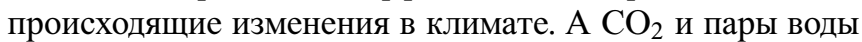
в значительной степени обусловливают данный эффект. Влажность атмосферы к тому же - один из важнейших метеопараметров атмосферы.

Возможности предлагаемого способа оценим на примере определения концентраций водяного пара и углекислого газа в атмосфере по спектральным измерениям поглощения в диапазоне 2640-2840 nm, в котором существует сильное перекрытие их линий поглощения.

Для получения главных компонент и коэффициентов регрессии было проведено моделирование процесса поглощения на основе данных из базы HITRAN, которая служит инструментом для расчета поглощения света в газовых средах, включая атмосферу [19]. Рассчитывались величины поглощения $I\left(\lambda_{i}\right)$ в диапазоне $2640-2840 \mathrm{~nm}$ с шагом $20 \mathrm{~nm}$ для случайных значений концентраций паров $\mathrm{H}_{2} \mathrm{O}$ и $\mathrm{CO}_{2}$ из диапазона от 0.1 до $5 \%$ для $\mathrm{H}_{2} \mathrm{O}$ и от 0.2 до $3 \%$ для $\mathrm{CO}_{2}$. Измерительная база задавалась длиной $10 \mathrm{~m}$.

При расчете моделируемой выборки $I\left(\lambda_{i}\right)$ для каждой температуры и давления учитывалось уширение спектральных линий. Используя полученные значения $I\left(\lambda_{i}\right)$, были выделены главные компоненты $C_{m}$ для каждой из температур и давления, равного $1 \mathrm{~atm}$. Объем каждой из выборок составлял 2000 состояний. Приведённые ниже результаты получены для двух главных компонент $C_{m}$, которые покрывают 99.9\% вариативности данных.

Численные значения следующих $C_{m i}$ главных компонент представляют собой линейную комбинацию из спектрального поглощения в интервале 2640-2840 nm с шагом $20 \mathrm{~nm}$ (для $n=10$ длин волн):

$$
C_{m}=\sum_{i=1}^{10} C_{m i} I\left(\lambda_{i}\right) .
$$

Множители $C_{m i}$ при спектральных величинах поглощения для главных компонент $C_{m}$ приведены в табл. 1 .

Далее, с использованием методов наименьших квадратов была установлена статистическая зависимость между главными компонентами $C_{m}$, коэффициентами $a, b_{m}$ и концентрациями компонент в смеси $M_{k, P, T}$. Коэффициенты $a, b_{m}$ регрессивного соотношения (6) для значений температур в интервале 253-293 K, соответствующие
Таблица 1. Множители $C_{m i}$ для главных компонент $C_{m}$ для задаваемых значений давления и температуры

\begin{tabular}{c|c|c}
\hline Длины волн, $\mathrm{nm}$ & $C_{1 i}$ & $C_{2 i}$ \\
\hline 2660 & 0.3218 & -0.2689 \\
2680 & 0.3263 & 0.2245 \\
2700 & 0.2504 & 0.6195 \\
2720 & 0.3285 & 0.1991 \\
2740 & 0.3155 & -0.3217 \\
2760 & 0.3336 & -0.1175 \\
2780 & 0.3122 & 0.3449 \\
2800 & 0.3348 & 0.0888 \\
2820 & 0.3152 & -0.3232 \\
2840 & 0.3151 & -0.3240
\end{tabular}

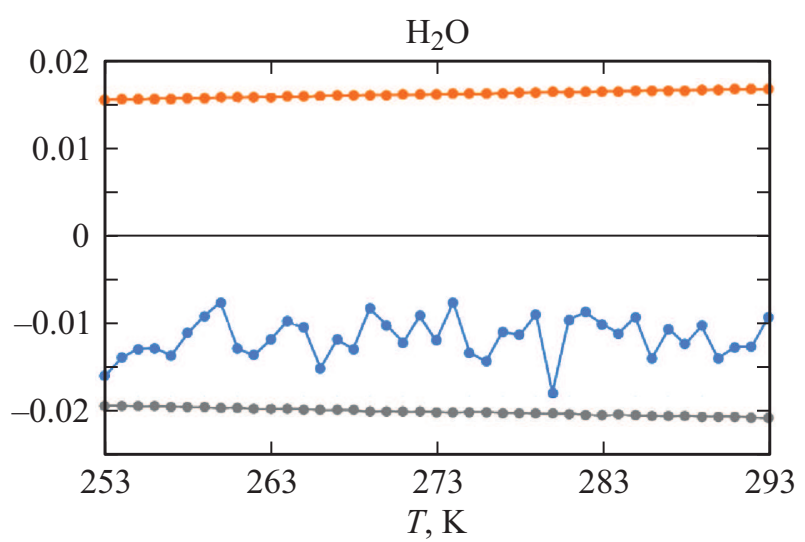

Рис. 1. Коэффициенты регрессивного соотношения (6) для значений температур в интервале $253-293 \mathrm{~K}$ ( $a$ - средняя кривая, $b_{1}-$ верхняя, $b_{2}-$ нижняя).

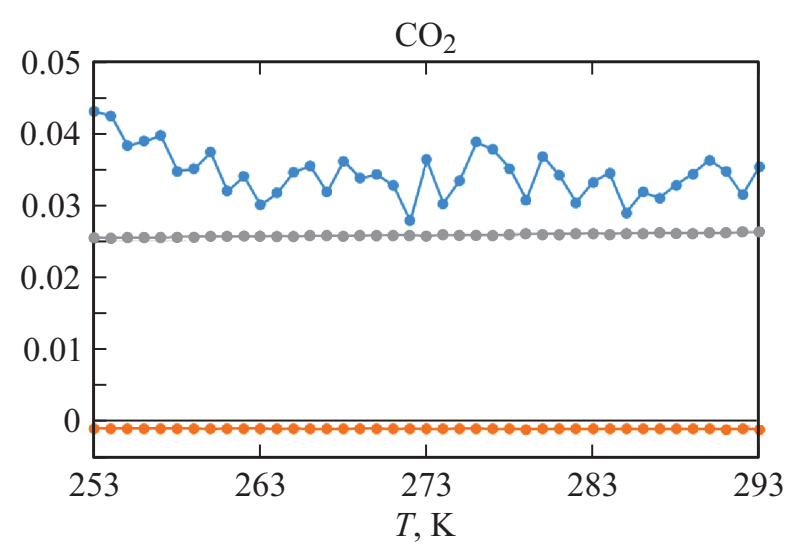

Рис. 2. Коэффициенты регрессивного соотношения (6) для значений температур в интервале $253-293 \mathrm{~K}$ ( $a$ - верхняя кривая, $b_{1}-$ нижняя, $b_{2}-$ средняя).

$\mathrm{H}_{2} \mathrm{O}$ и $\mathrm{CO}_{2}$, приведены на рис. 1 и 2 (по оси абсцисс отложены значения температуры $T$ ).

Оценка погрешности восстановления концентраций паров $\mathrm{H}_{2} \mathrm{O}$ и $\mathrm{CO}_{2}$ была проведена на новой выборке $I\left(\lambda_{i}\right)$ при наложении на каждое значение из нее по- 
Таблица 2. Погрешности (в \%) определения концентраций паров $\mathrm{H}_{2} \mathrm{O}$ и $\mathrm{CO}_{2}$

\begin{tabular}{c|c|c|c}
\hline$T \mathrm{~K}$ & $\begin{array}{c}\text { Погрешность } \\
\text { восстановления } \\
\text { для молекул } \mathrm{H}_{2} \mathrm{O}, \\
\text { метод регрессионных } \\
\text { соотношений }\end{array}$ & $\begin{array}{c}\text { Погрешность } \\
\text { восстановления } \\
\text { для молекул } \mathrm{CO}_{2}, \\
\text { метод регрессионных } \\
\text { соотношений }\end{array}$ & $\begin{array}{c}\text { Погрешность } \\
\text { восстановления для } \\
\text { молекул СО }, \text { метод } \\
\text { дифференциального } \\
\text { поглощения }\end{array}$ \\
\hline 253 & 1.84 & 3.94 & 83.03 \\
254 & 1.86 & 3.91 & 83.76 \\
255 & 1.94 & 3.72 & 83.74 \\
280 & 1.94 & 3.89 & 81.56 \\
281 & 1.94 & 3.65 & 81.59 \\
293 & 1.99 & 3.63 & 83.19 \\
291 & 1.96 & 3.68 & 83.07 \\
293 & 1.94 & 3.82 & 82.41 \\
\end{tabular}

Таблица 3. Коэффициенты линейной регрессии (для задаваемого диапазона значений давления и температуры)

\begin{tabular}{c|r|r}
\hline & $\mathrm{H}_{2} \mathrm{O}$ & $\mathrm{CO}_{2}$ \\
\hline$a$ & -0.004 & 0.0368 \\
$b_{1}$ & 0.0161 & -0.0010 \\
$b_{2}$ & -0.0200 & 0.0258
\end{tabular}

грешности 5\% (объем выборки - 500). Задаваемые значения концентраций паров $\mathrm{H}_{2} \mathrm{O}$ и $\mathrm{CO}_{2}$ сравнивалась с рассчитываемыми концентрациями по (6). Значения погрешностей для концентраций паров $\mathrm{H}_{2} \mathrm{O}$ и $\mathrm{CO}_{2}$ (для начала, середины и конца диапазона температур) приведены в табл. 2. Как видно из таблицы, погрешности восстановления составили для паров $\mathrm{H}_{2} \mathrm{O}$ и $\mathrm{CO}_{2}$ соответственно менее 2 и $4 \%$, что говорит об устойчивости регрессионных соотношений к погрешностям измерения величин поглощения $\sim 5 \%$.

Оценивалась погрешность восстановления концентрации $\mathrm{CO}_{2}$ с использованием указанного выше диапазона длин волн и по методу дифференциального поглощения как наиболее точному и устойчивому из известных. Однако даже при использовании наиболее слабого перекрытия спектральных линий в данном диапазоне длин волн при определении концентрации $\mathrm{CO}_{2}$ по данному методу в реальной атмосфере погрешности составляют более $80 \%$ (табл. 2).

\section{Оценка эффективности метода для задаваемого диапазона значений давления и температуры}

Выше численные значения $a, b_{m}, C_{m}$ устанавливали для каждой пары величин давления $P(P=1 \mathrm{~atm})$ и температуры $T$ и связывали концентрации компонентов газовой смеси с главными компонентами вектора измерений $I\left(\lambda_{i}\right)$ для ситуации, когда измерения проводятся при известных значениях температуры и давления. Представляет практический интерес проводить измерения и в условиях, не требующих знания величин давления $P$ и температуры $T$ исследуемой газовой смеси. Численное значение $a, b_{m}, C_{m}$ для (6) и в этом случае устанавливаются на основе измерений или численного расчета $I\left(\lambda_{i}\right)$ для множества ситуаций или моделирующих его фантомов с известными значениями концентраций $M_{k}$ компонент газовой смеси при различных значениях ее температуры и давления, но уже в задаваемом диапазоне их значений (например, для времени года, для возможных значений в технологических процессах и т.д.).

Однако если для рассмотренной выше ситуации используется вектор реализаций $\mathbf{I}\left(\lambda_{i}\right)$, соответствующий разным значениям $M_{k}$ для конкретных (измеренных) значений давления $P$ и температуры $T$, то в рассматриваемом случае вектор реализаций I включает все значения $I\left(\lambda_{i}\right)$, соответствующие разным значениям $M_{k}$ для любых значений $P$ и $T$ из задаваемого диапазона их разброса (задается диапазон изменений $M_{k}, P$ и $T$ ).

После получения векторов $\mathbf{I}\left(\lambda_{i}\right)$ и среднего вектора $\mathbf{I}\left(\lambda_{i}\right), V$, а также вышеотмеченного аналитического выражения (6) они могут применяться для определения концентраций компонентного состава газовой смеси по измеряемым значения $I\left(\lambda_{i}\right)$. При этом не требуется знание температуры и давления газовой смеси.

Возможности предлагаемого способа в ситуациях, не требующих знания величин давления и температуры исследуемой газовой смеси, оценивались для определения водяного пара и углекислого газа в атмосфере по спектральным измерениям поглощения в диапазоне 2640-2840 nm, в котором существует сильное перекрытие их линий поглощения (для тех же условий, что и для атмосферных ситуаций, когда давления и температуры фиксируются). Коэффициенты линейной регрессии для 
значений температур в интервале $253-293 \mathrm{~K}$ и давления $1 \mathrm{~atm}$ приведены в табл. 3.

Значения погрешностей определения концентраций $\mathrm{CO}_{2}$ и паров $\mathrm{H}_{2} \mathrm{O}$ в атмосфере при измерениях, когда величины давления и температуры не устанавливаются, составляет соответственно 6.1 и $3.6 \%$.

\section{Заключение}

Таким образом, для определения количественных значений концентрации каждой компоненты газовой смеси предлагаемым методом не требуется сравнений результатов измерения с функцией, описывающей связь (расчет) регистрируемых сигналов с параметрами исследуемой среды, использования калибровочных сравнительных моделей, устанавливаемых заранее эмпирически (экспериментально), методов решения некорректных обратных задач. В предлагаемом методе требуется заранее (только один раз) путем численного моделирования исследуемой среды (расчета величины спектрального поглощения) установить коэффициенты регрессионного соотношения между концентрацией и главными компонентами величины спектрального поглощения для каждой компоненты среды и использовать его для данной среды в разных условиях (температура, давление; причем их можно устанавливать как для фиксированных значений температуры и давления, так и для задаваемого диапазона значений их значений). При расчете величины поглощения учитываются все перекрытия спектральных линий с учетом их уширения при разных температурах и давлении, что позволяет использовать метод в многокомпонентных средах с сильно перекрывающимися спектральными линиями. Более того, устанавливаемые регрессионные соотношения устойчивы к погрешностям измерительной информации, поскольку при получении их эти погрешности накладываются на рассчитываемые величины поглощения. Все это приводит к повышению точности предлагаемого метода определения компонентного состава газовой среды. К тому же позволяет определять их оперативно (в реальном масштабе времени), поскольку расчет концентраций с использованием устанавливаемых связей (аналитических выражений) между определяемыми и измеряемыми величинами не представляет вычислительных сложностей, не требует отбора проб, так как может использоваться как в базисном, так и в дистанционном вариантах реализации.

\section{Конфликт интересов}

Авторы заявляют, что у них нет конфликта интересов.

\section{Список литературы}

[1] Фираго В.А., Манак И.С., Вуйцик В. Контроль газового состава сред методами лазерной и светодиодной абсорбционной спектроскопии. Минск: Акад. Упр. при Президенте Республики Беларусь, 2006. 303 с.
[2] Межсерис Р. Лазерное дистанционное зондирование. М.: Мир, 1987. $550 \mathrm{c.}$

[3] Городничев B.A. Разработка методов и оптикоэлектронных средств лазерного оперативного контроля многокомпонентных газовых смесей составляющих ракетных топлив и других токсичных веществ. Автореф. докт. дис. 2009. 308 с.

[4] Little J.P. et al. Patent US 8,686,364 B1, 2014.

[5] Катаев М.Ю., Лукьянов А.К., Бекетов А.А. // Журнал Сибирского федерального университета. Техника и технологии. 2018. 11(1). С. 77. doi 10.17516/1999-494X-0011

[6] Катаев М.Ю. // Доклады ТУСУРа. 2015. № 4 (38). С. 155.

[7] Тихонов А.Н., Арсенин В.Я. Методы решения некорректных задач. М.: Наука, 1979. 288 с.

[8] Козлов В.Л., Кугейко М.М. Патент РБ № 12455, 2007.

[9] Васильев А.В., Мельникова И.Н. Методы прикладного анализа результатов натурных измерений в окружающей среде. СПб.: Балт. гос. техн. ун-т., 2009. 369 с.

[10] Wang L., Jacques S., Zheng L. // Computers Methods and Programs in Biomedicine. 1995. N 47. P. 131.

[11] Zonios G., Dimou A. // Optics Express. 2006. V. 14. N 19. P. 8661.

[12] Anderson R.R., Perrish J.A. // J. Invest. Dermatol. 1981. V. 77. P. 13.

[13] Farrell T.J., Patterson M.S., Wilson B.C. // Med. Phys. 1992. V. 19. N 4. P. 879.

[14] Синичкин Ю.П., Ути, С.Р., Пилипенко Е.А. // ОПт. и спектр. 1996. Т. 80. № 2. С. 260.

[15] Барун В.В., Иванов А.П. // Квант. электрон. 2010. Т. 40. № 4. C. 371.

[16] Кугейко М.М., Лысенко С.А. Лазерная спектронефелометрия аэродисперсных сред. Минск: БГУ, 2012. 208 с.

[17] Лазерный контроль атмосферы / Под. ред. Хинкли Э.Д. М: Мир, 1979. $416 \mathrm{c.}$

[18] Колемаев В.А., Калинина В.Н. Теория вероятностей и математическая статистика. М.: ИНФРА-М, 1997. 300 с.

[19] Кочанов Р.В. и др. // Журн. количественной спектроскопии и переноса излучения. 2016. Т. 177. № 7. С. 15. 